\title{
25 Research Square \\ Ovarian Gonadoblastoma with the Karyotype of 46, XX: A Case Report
}

Fengju Zhao

Gansu Provincial Hospital

Yingchao Zhao

Tongji Medical College of Huazhong University of Science and Technology: Huazhong University of Science and Technology Tongji Medical College

\section{Biyuan Xing}

Tongji Medical College of Huazhong University of Science and Technology: Huazhong University of Science and Technology Tongji Medical College

Zhao Liu ( $\square$ liuzhao_cc@126.com)

Huazhong University of Science and Technology Tongji Medical College

\section{Case report}

Keywords: gonadoblastoma, dysgerminoma, FoxO, KIT

Posted Date: August 20th, 2021

DOl: https://doi.org/10.21203/rs.3.rs-783535/v1

License: (c) (i) This work is licensed under a Creative Commons Attribution 4.0 International License. Read Full License 


\section{Abstract}

Gonadoblastoma is a rare tumor comprised of sex cord derivatives and germ cells. The risk for developing gonadoblastoma increases significantly in patients who possess a $Y$ chromosome or $Y$ chromosome material. A 49-year-old Chinese woman found a pelvic mass during a routine physical examination. Pathological analysis after surgery indicated that the tumor was unilateral ovarian gonadoblastoma with dysgerminoma. Compared with other cases in the literature, our patient was the oldest, and the tumor mass was smaller. Karyotype analysis of peripheral blood lymphocytes revealed that the woman had a 46, XX female karyotype. Whole-exon sequencing revealed that some mutations, such as altered somatic genes in the Forkhead box protein $\mathrm{O}$ (FoxO) signaling pathway and KIT, might cause the disease. In conclusion, we described a rare case of gonadoblastoma in a woman who had normal routine menstruation, sexual development, and successful pregnancies and possessed a normal female 46, XX karyotype.

\section{Introduction}

First reported by Scully RE in 1953, gonadoblastoma is a rare tumor comprised of sex cord derivatives and germ cells in a nested pattern surrounded by the ovarian stroma ${ }^{1}$. Gonadoblastomas are considered carcinomas in situ, while the germ cell components in $50 \%$ of patients overgrow and can transform into malignant germ cell tumors. The most common progression is the transformation to dysgerminoma and occasionally to other germ cell tumors, such as yolk sac tumors, embryonal carcinomas, and choriocarcinomas ${ }^{2}$. Most patients with gonadoblastoma have a 46, $X Y$ karyotype or some form of $Y$ chromosome mosaicism, such as $45, X / 46, X Y$ mosaicism2. Rare gonadoblastoma cases with the normal female $46, \mathrm{XX}$ karyotype have been report ${ }^{3-14}$.

In this report, we describe a case of gonadoblastoma mixed with dysgerminoma in a 49-year-old woman who had a normal 46, XX karyotype. Her menstruation, sexual development and reproduction were normal. Moreover, whole-exon sequencing revealed certain suspectable mutations, such as KIT.

\section{Case Report}

The patient was a 49-year-old woman, Each Asian race, gravidity three, parity one, who had attained menarche at the age of 12 and had regular 28-day menstrual cycles lasting for five days before entering menopause in 2012. At the age of 42 , she was diagnosed with invasive ductal carcinoma of the left breast (pT2NOMO, stage IIA). Then, she underwent modified radical mastectomy for the left breast and received tamoxifen for five years post-surgery. In January 2018, the patient at the age of 49 , a pelvic mass was found during a routine physical examination. The patient had no uncomfortable symptoms. Computed tomography showed a round-like, well-defined, low density, mildly enhancing lesion measuring $5.6 \mathrm{~cm} \times 3.9 \mathrm{~cm} \times 5.0 \mathrm{~cm}$ directly behind the uterus. The detection of tumor markers revealed that the CA125 level was $43.3 \mathrm{IU} / \mathrm{mL}$, while $\beta-\mathrm{HCG}$ and other tumor marker levels were within the normal range. 
This patient underwent subsequent laparoscopic surgery, including total hysterectomy, bilateral salpingooophorectomy, pelvic lymphadenectomy, paraaortic lymphadenectomy, partial omentum resection, and appendicectomy. During laparoscopic surgery, the right ovary was observed with an abnormally enlarged morphology. This involved a solid mass contained in an intact capsule. Pathological analysis indicated that the tumor was a dysgerminoma involving the surface of the right ovary with a small amount of the gonadoblastoma component regionally. The patient was diagnosed with stage IC ovarian gonadoblastoma and dysgerminoma. After the operation, the patient received four cycles of combination chemotherapy containing bleomycin, etoposide, and cisplatin. No recurrence was found during 31 months of regular follow-up after chemotherapy. Written informed consent was obtained from the patient for publication of this case report and any accompanying images.

The dysgerminoma was comprised of large and identical tumor cells separated by collagen fiber bands, and tumor nuclei were similar in size. The nucleoli were obvious, even during the stage of karyomitosis (Fig. 1a). The gonadoblastoma was arranged in the form of nests, which were comprised of germ cells mixed with sexual cord cells similar to immature Sertoli cells and granulocytes. The germ cells were round with transparent cytoplasm and large, round nuclei. The sex cord cells were small and deeply stained, the nuclei were slender, and the nucleoli were not obvious (Fig. 1g). Immunohistochemical analysis indicated that the dysgerminoma component had positive staining for D2-40 (Fig. 1b), OCT3/4 (Fig. 1C), PLAP (Fig. 1d), SALL4 (Fig. 1e), and CD117 (Fig. 1f) and negative staining for a-inhibin, Calretinin and EMA. In the gonadoblastoma component, the sex cord cell component showed positive staining for a-inhibin (Fig. 1h) and Calretinin (Fig. 1i), while the germ cell component indicated positive staining for SALL4 (Fig. 1j), PLAP (Fig. 1k), and OCT3/4 (Fig. 1I). These features supported the diagnosis of gonadoblastoma.

Karyotype analysis of peripheral blood lymphocytes revealed that the woman had a 46, XX female karyotype (Fig. 3). Genomic DNA was extracted from both paraffin-embedded sections and peripheral blood according to standard procedures. The primary paraffin-embedded tissues were dewaxed in xylene and rehydrated in ethanol and then positioned in proteinase $\mathrm{K}$ buffer before DNA extraction. Then DNA was fragmented, exome-enriched, and sequenced. Whole-Exon Sequencing (WES) was conducted at BGI with 170x sequencing depth for the peripheral blood sample and $\sim 428 \mathrm{x}$ sequencing depth for the paraffin-embedded sample, respectively. After removing the adaptors and low-quality reads (Phred score $<15)$ produced by the sequencing process, clean reads were further mapped to the human reference genome assembly GRCh19 using the Burrows-Wheeler-Alignment (BWA) tool. Duplicate reads were removed by Picard. Small mutations were called and filtered respectively by GATK4 for both the sequenced samples. Germline mutations were obtained from the peripheral blood sample directly. Somatic mutations were obtained by filtering out the germline mutations from the mutations called in paraffin-embedded sections. Both germline mutations and somatic mutations were annotated by ANNOVAR. Public database, including the latest 1000 Genomes Project dataset (1KG), Exome Aggregation Consortium dataset (EXAC), gnomAD dataset (gnomAD), the COSMIC cancer database and the Clinvar database were used in the annovation. We filtered both somatic mutations and germline mutations by the following rules: 1 ) variants beside exonic or splicing region were filtered out; 2 ) variants 
annotated as synonymous SNV or unknown were filtered out; 3) variants that were reported in one of the $1 \mathrm{KG}$, ExAC or gnomAD with allele frequency equal or greater than $1 \%$ were filtered out; 4 ) somatic variants with allele frequency smaller than $5 \%$ were filtered out. After filtering, 84 variants were left for somatic mutations, and 516 variants were left for germline mutations. We subsequently obtain pathogenic variants from germline mutations and somatic mutations separately. For the germline mutations: mutations with pathogenic clinical significance annotated by Clinvar were remained. For the somatic mutations: insertions, deletions and splicing variants were remained as they affect the amino acid sequences of the proteins. We predict the pathogenicity of the single nucleotide variants by SIFT, Polyphen2 (HDIV/HVAR), LRT, MutationTaster, MutationAssessor, MetaSVM/LR, M-CAP, PROVEAN, FATHMM and FATHMM-MKL. Variants annotated as pathogenic or like pathogenic by at least three software were remained for further analysis. Thirty-nine variants, with four germline mutations (i.e., GJB2, SPINK5, SMPD1, CEP290) and 34 somatic mutations (e.g., KIT, USP6, NANOG) left marked as deleterious variants as shown in Table 1. Figure 2 demonstrates the distribution of the variants on the human genome.

Gene Ontology (GO) and Kyoto Encyclopedia of Genes and Genomes (KEGG) pathway analyses were performed for germline mutations and somatic mutations separately (Fig. 4). The top 10 enriched cellular component, biological process and molecular function in GO and the top $30 \mathrm{KEGG}$ pathways were displayed. Somatically altered genes showed significant enrichment for sex differentiation and development of the first sex character (Fig. 4a). This result may confirm the tissue origin of this tumor, which originates from germ cells. Notably, KIT was implicated in the differentiation of germ cells. In addition, somatic mutated genes were mainly enriched in the dysregulation of transcriptional activity and DNA replication, which might be the putative triggering factors. Results of the KEGG analysis showed that somatic mutated genes were enriched in signaling pathways related to cell proliferation and differentiation, such as the Forkhead box protein O (FoxO) signaling pathway, EGFR tyrosine kinase inhibitor resistance, the ERBB2 signaling pathway, PI3K-Akt signaling pathway, MAPK signaling pathway, homologous recombination deficiency and mRNA surveillance (Fig. 4b). KIT, encoding a receptor tyrosine kinase, is involved in mitogen-activated protein kinase (MAPK) and PI3K-Akt signaling pathways. No remarkable GO and KEGG enrichment were observed for the germline mutated genes (Fig. 4c and d).

\section{Discussion}

Gonadoblastoma is considered rare cancer with a real incidence that is poorly characterized in the literature. It most frequently affects young women, in which gonadal dysgenesis appearing as virilization can be frequently observed. Most cases of gonadoblastoma present with $\mathrm{Y}$ chromosome material, especially sex-determining region $\mathrm{Y}$ (SRY) detection, while the feminine phenotype is typically rare, accounting for only $8 \%$ of cases. ${ }^{2}$ The karyotype analysis of our case demonstrated a normal $46, \mathrm{XX}$ karyotype without $Y$ chromosome material. The patient had normal menstruation, normal sexual development and even successful pregnancies, which eliminated the possibility of gonadal dysgenesis. 
There have been rare cases of gonadoblastoma with the $46, X X$ karyotype reported in the literature. We performed a review of the literature with PubMed for articles focusing on gonadoblastoma with the 46, XX karyotype and found 14 cases reported between 1990 and 2020. Twelve cases from source journals of SCl were selected. The 12 patients were females younger than 40 years old with the $46, X X$ karyotype. Tumor sizes in most reported cases $(7 / 12)$ were larger than $10 \mathrm{~cm}$. Our case report the oldest patient with the smallest tumor size $5.6 \mathrm{~cm}$ in diameter, which was unusually small. The somatic gene mutation of our case showed a correlation with abnormal sex development, while the germline mutation did not. Somatic gene mutations occur later than germline mutations; this might explain the older age and smaller tumor volume of our case.

In our review, most of the cases received chemotherapy (8 of 12). Partly due to the young age and favorable prognosis, four newly diagnosed patients underwent salpingo-oophorectomy of the tumorinvolved side ${ }^{7-10}$, three patients underwent single-sided oophorectomy ${ }^{3,4,11}$, and two patients underwent a bilateral operation. ${ }^{5,6}$ But for earlier patients, three cases diagnosed before 2010 underwent a hysterectomy ${ }^{12-14}$. For gonadoblastoma patients with dysgenetic gonads, the recommended treatment usually is bilateral oophorectomy and hysterectomy. However, in patients with the $46, X X$ karyotype, it is more difficult to determine the appropriate treatment due to the rarity of the disease and the uncertainty of the necessity for bilateral gonadectomy ${ }^{4}$. In our case, we performed both hysterectomy and bilateral salpingo-oophorectomy. This approach may be especially suitable for older females without the need to preserve fertility. Therefore, we recommend that a different surgical approach should be appropriate for specific patients. As the disease occurs more frequently in young females and the prognosis appears to be favorable, the approach that preserves fertility sometimes may be more suitable.

Although the patient had dual cancer of the ovary and breast, she was not a typical hereditary breast cancer-ovarian cancer patient. She did not have a BRCA mutation, and the pathological type was not a typically serous carcinoma. Germline alterations were observed in the genes involved in the MLL3/4 signaling pathway (GO) and phospholipase D signaling pathway (KEGG). These findings gave an indication that the chromosome remodeling induced an epigenetic effect coupled with the activation of an intracellular signaling pathway, which may serve as the initial step of oncogenesis. Somatic alterations were observed in genes enriched in the FoxO signaling pathway. FoxO genes regulate critical cellular functions (apoptosis, cell cycle arrest, DNA repair and female reproduction) that are dysregulated in cancer cells. An increasing number of studies have also indicated a close relationship between the development of gonadoblastoma and genes regulating sexual determination and differentiation (e.g., KIT, SRY, WT1, SOX9, FoxI2, and TSPY) ${ }^{15}$. KIT belongs to the Type III tyrosine kinase receptor family, of which activating mutations have been found in human ovarian dysgerminomas. Spontaneous KIT mutation(s) lead to increased survival and proliferation of undifferentiated oogonia ${ }^{16}$. This might be caused by activating the MAKP and PI3K-Akt pathways. Previous research mainly focused on TSPY and underlined the importance of the presence of (part of) the Y-chromosome in the development of $\mathrm{GB}^{17}$. Our study highlights the potential of the KIT to be a candidate for gonadoblastoma mutation sites in $46, X X$ females. 


\section{Conclusion}

In this study, we described a rare case of gonadoblastoma in a woman who had normal routine menstruation, sexual development, and even successful pregnancies and possessed a normal female 46, XX karyotype. Compared with other cases in the literature, our patient was the oldest, and the tumor mass was smaller. Whole-exon sequencing revealed that somatically altered genes of the FoxO signaling pathway and $K I T$, not germline alterations, may play roles in the proliferation and differentiation of germ cells.

\section{Declarations}

\section{Ethics approval and consent to participate}

Not applicable.

\section{Consent for publication}

Not applicable.

\section{Availability of data and materials}

The data used to support the findings of this study are available from the corresponding author upon request.

\section{Competing interests}

The authors declare that they have no competing interests.

\section{Funding}

N.A.

\section{Authors' contributions}

LZ designed the study and provided clinical information. ZFJ and ZYC analyzed the results of Sanger sequencing, bioinformatic and in vitro study. XBY wrote the manuscript and ZYC revised it. All authors read and approved the final manuscript.

\section{Acknowledgements}

N.A.

\section{References}


1. Scully RE. Gonadoblastoma; a gonadal tumor related to the dysgerminoma (seminoma) and capable of sex-hormone production. Cancer 1953; 63: 455-463.

2. Scully RE. Gonadoblastoma. A review of 74 cases. Cancer 1970; 25: 1340-1356.

3. McCuaig JM, Noor A, Rosen B et al. Case report: use of tumor and germline $Y$ chromosomal analysis to guide surgical management in a $46, X X$ female presenting with gonadoblastoma with dysgerminoma. Int J Gynecol Pathol 2017; 36: 466-470.

4. Esin S, Baser E, Kucukozkan T, Magden HA. Ovarian gonadoblastoma with dysgerminoma in a 15year-old girl with 46, XX karyotype: case report and review of the literature. Arch Gynecol Obstet 2012; 285: 447-451.

5. Yilmaz B, Gungor T, Bayramoglu H, Soysal S, Mollamahmutoglu L. Bilateral ovarian gonadoblastoma with coexisting dysgerminoma in a girl with 46, XX karyotype. J Obstet Gynaecol Res 2010; 36: 697700 .

6. Obata NH, Nakashima N, Kawai M, Kikkawa F, Mamba S, Tomoda Y. Gonadoblastoma with dysgerminoma in one ovary and gonadoblastoma with dysgerminoma and yolk sac tumor in the contralateral ovary in a girl with 46XX karyotype. Gynecol Oncol 1995; 58: 124-128.

7. Roth LM, Davis MM, Czernobilsky B. Classic and "dissecting" gonadoblastoma in a phenotypic girl with a 46, XX peripheral karyotype and no evidence of a disorder of sex development. Int J Gynecol Pathol 2018; 38: 581-587.

8. Arafah MA, Raddaoui LE. Malignant mixed germ cell tumor overgrowing a gonadoblastoma in a female with a 46, XX karyotype: a case report. Int J Surg Patho/ 2018; 26: 287-292.

9. Gru AA, Williams ES, Cao D. Mixed gonadal germ cell tumor composed of a spermatocytic tumor-like component and germinoma arising in gonadoblastoma in a phenotypic woman with a $46, \mathrm{XX}$ peripheral karyotype. Am J Surg Pathol 2017; 41: 1290-1297.

10. Koo YJ, Chun YK, Kwon YS et al. Ovarian gonadoblastoma with dysgerminoma in a woman with 46XX karyotype. Pathol Int 2011; 61: 171-173.

11. Erdemoglu E, Ozen S. Ovarian gonodoblastoma with yolk sac tumor in a young 46, XX female: case report. Eur J Gynaecol Oncol 2007; 28: 516-518.

12. Erhan Y, Toprak AS, Ozdemir N, Tiras B. Gonadoblastoma and fertility. J Clin Pathol 1992; 45: 828829.

13. Gorosito M, Pancera B, Sarancone S, Nocito AL. Gonadoblastoma: an unusual ovarian tumor. Ann Diagn Pathol 2010; 14: 247-250.

14. Zhao S, Kato N, Endoh Y, Jin Z, Ajioka Y, Motoyama T. Ovarian gonadoblastoma with mixed germ cell tumor in a woman with 46, XX karyotype and successful pregnancies. Pathol Int 2000; 50: 332-335.

15. Kersemaekers AM, Honecker F, Stoop H et al. Identification of germ cells at risk for neoplastic transformation in gonadoblastoma: an immunohistochemical study for OCT3/4 and TSPY. Hum Pathol 2005; 36: 512-521. 
16. Hoei-Hansen C E, Kraggerud S M, Abeler V M, et al. Ovarian dysgerminomas are characterised by frequent KIT mutations and abundant expression of pluripotency markers[J]. Molecular Cancer 2007, 6(1): 1-12.

17. Hersmus R, Stoop H, Van De Geijn G J, et al. Prevalence of c-KIT mutations in gonadoblastoma and dysgerminomas of patients with disorders of sex development (DSD) and ovarian dysgerminomas[J]. PloS one, 2012, 7(8): e43952.

\section{Figures}



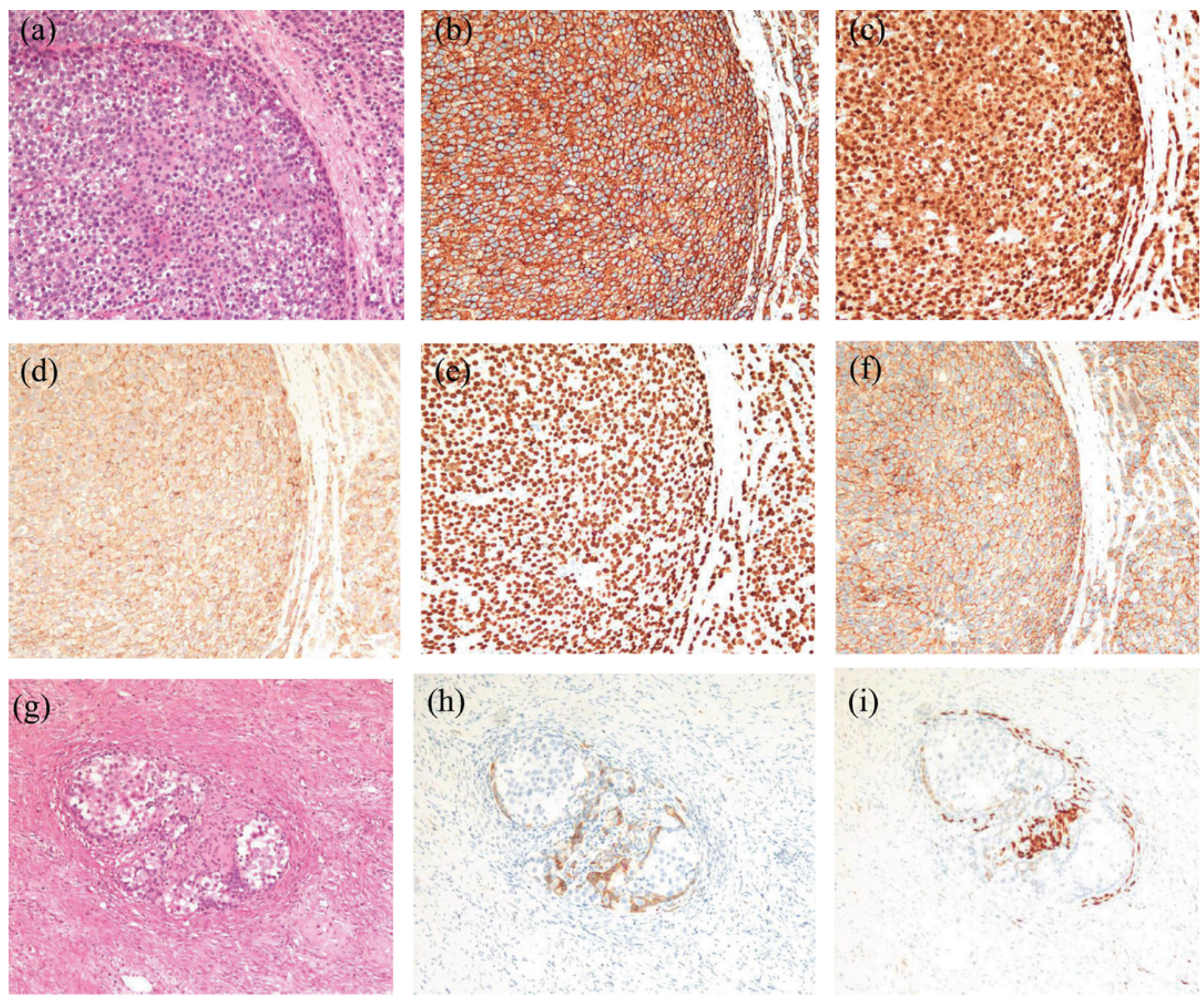

(h)

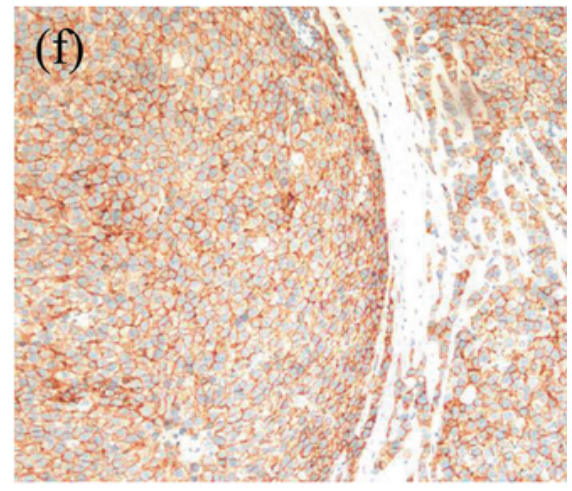

(j)

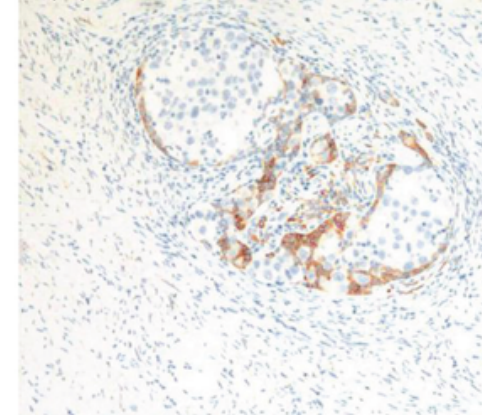

(i)

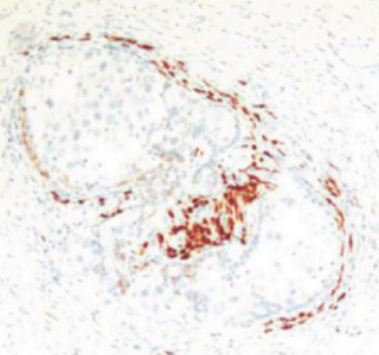

(k)

(1)
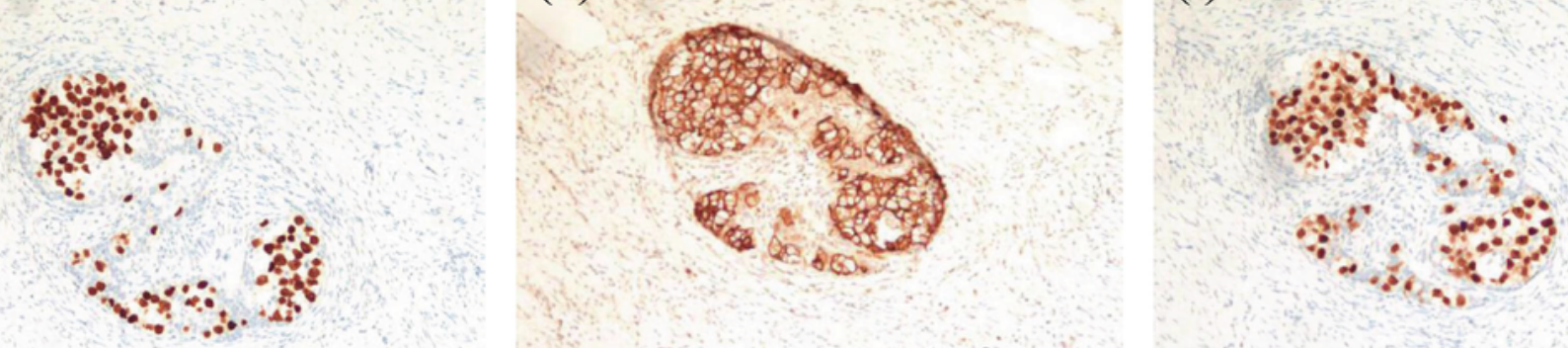

\section{Figure 1}

Hematoxylin and eosin (H-E) and immunohistochemical staining (a) H-E staining of the dysgerminoma component (original magnification $\times 100$ ), (b) The cytoplasm and cell membrane were positive for D2-40 (original magnification $\times 100$ ), (c) The nucleus was positive for OCT3/4 (original magnification $\times 100$ ), (d) The cell membrane was positive for PLAP (original magnification $\times 100$ ), (e) The nucleus was positive for SALL4 (original magnification $\times 100$ ), (f) The cytoplasm and cell membrane were positive for CD117 
(original magnification $\times 100$ ), (g) $\mathrm{H}-\mathrm{E}$ staining of the gonadoblastoma component (original magnification $\times 100$ ), (h) The cytoplasm of the sex cord component was positive for a-inhibin (original magnification $\times 100$ ), (i) The cytoplasm and nucleus of the sex cord component were positive for Calretinin (original magnification $\times 100)$, (j) The nucleus of the germ cell component was positive for SALL4 (original magnification $\times 100)$, $(k)$ The cell membrane of the germ cell component was positive for PLAP (original magnification $\times 100$ ), (I) The nucleus of the germ cell component was positive for OCT3/4 (original magnification $\times 100$ ).

\section{chr1}

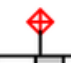

chr2

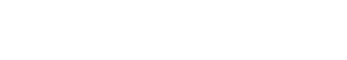

chr3

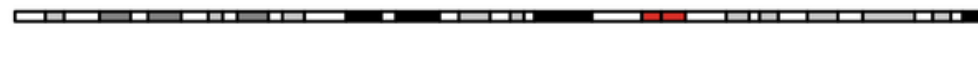

chr4

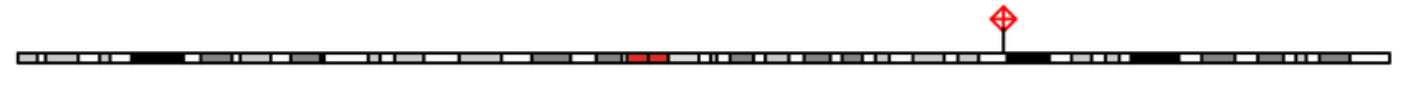

chr5

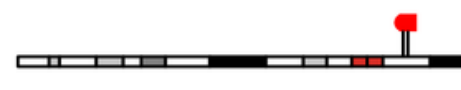
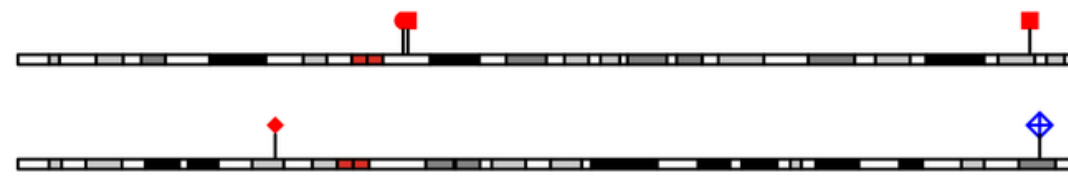

\section{chr6}

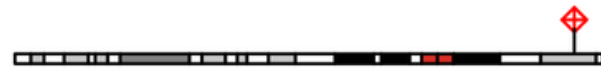

\section{chr7}

chr8

chr9

chr11

19.

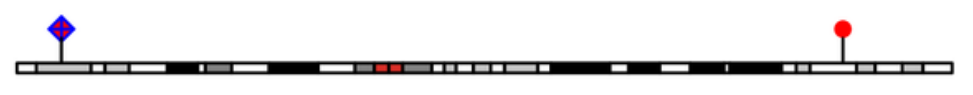

chr12

i.
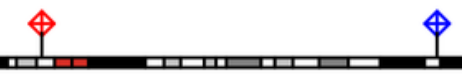

chr13

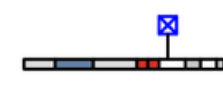

chr14

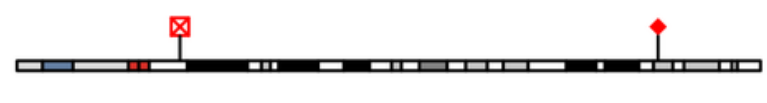

chr15

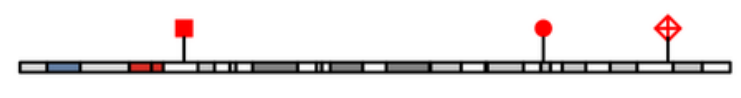

chr17
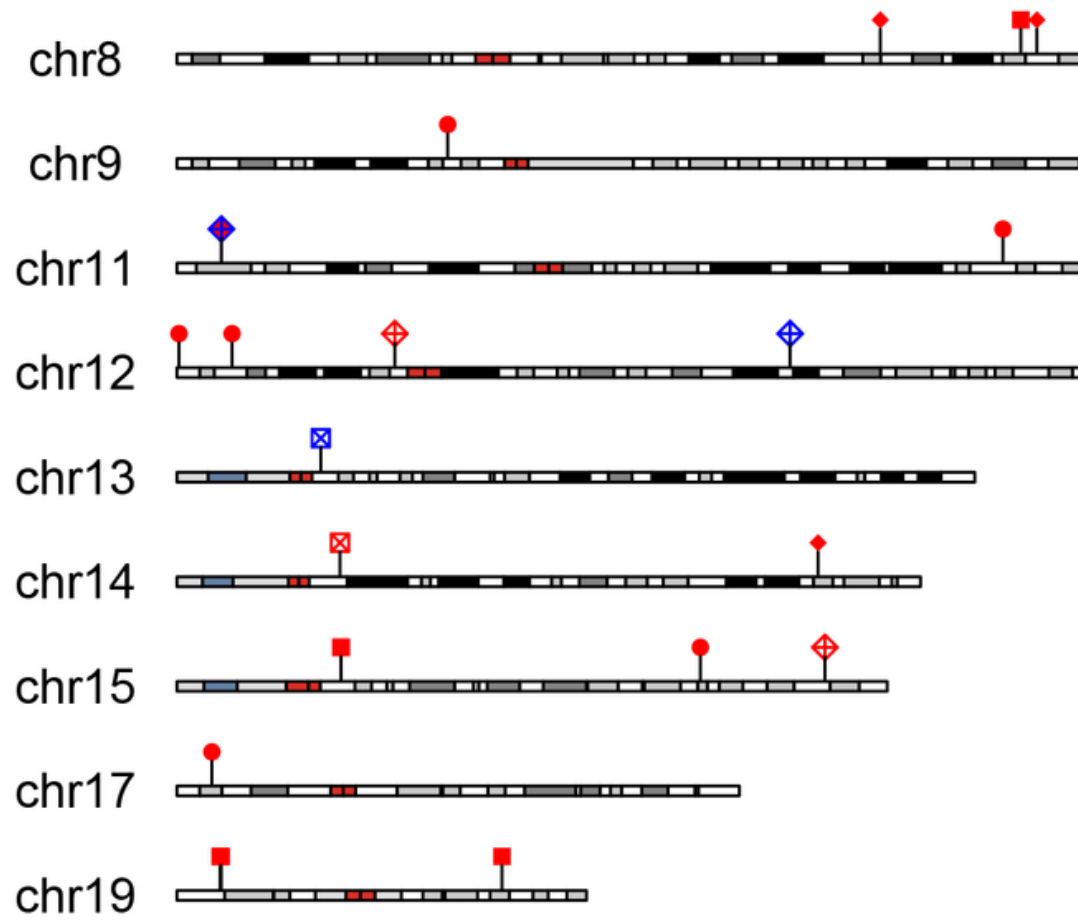

chr20

시․

chr22

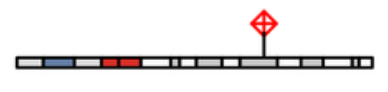

chrX

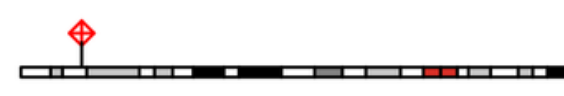

- Nonsynonymous SNV

- Nonframeshift Deletion

$\Delta$ Frameshift Insertion

$\diamond$ Frameshift Deletion

- Splicing

- Somatic Mutation

- Germline Mutation 
Figure 2

The overview of 39 deleterious variants on chromosomes
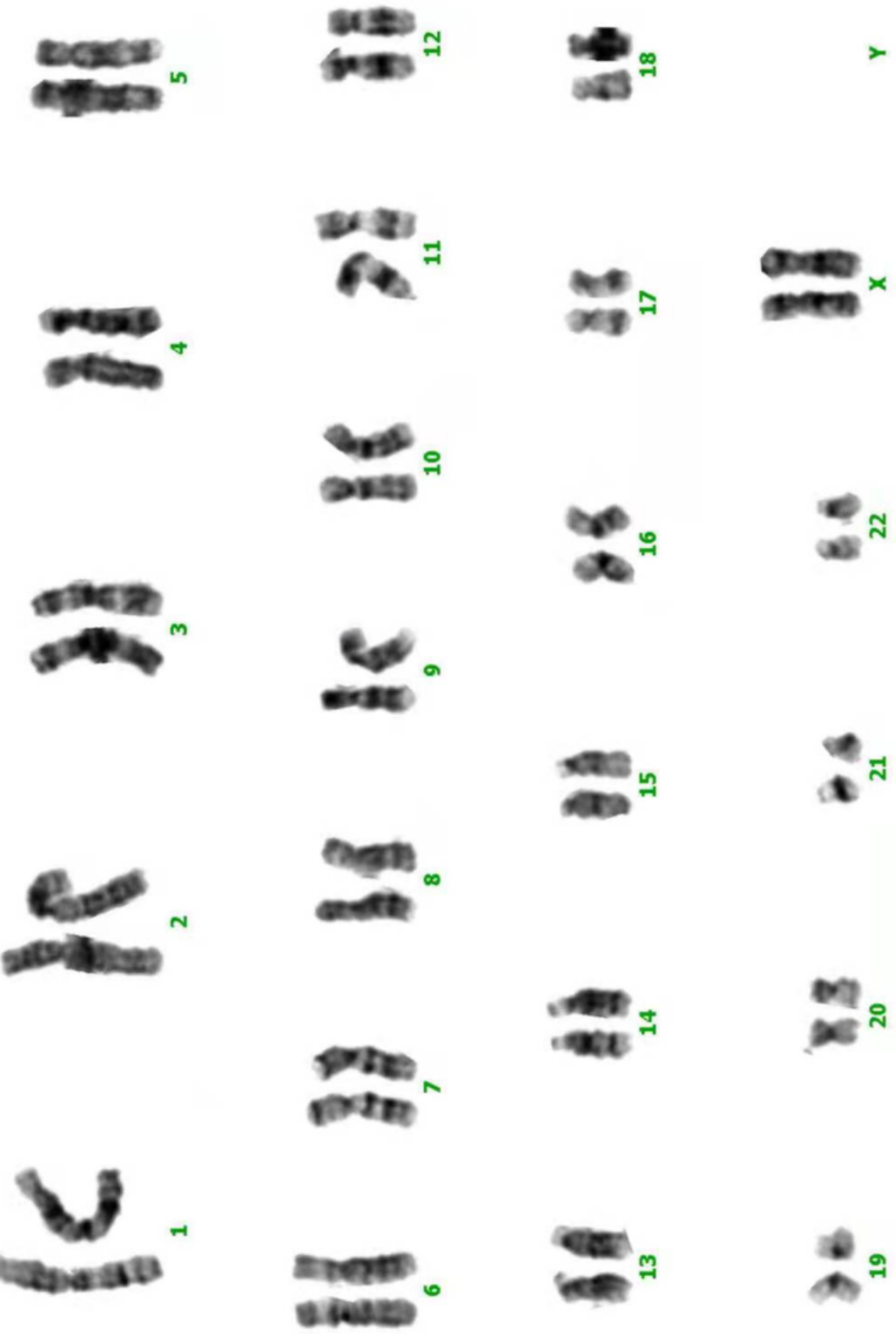

\section{Figure 3}

Karyotype analysis of peripheral blood lymphocytes from the patient. 

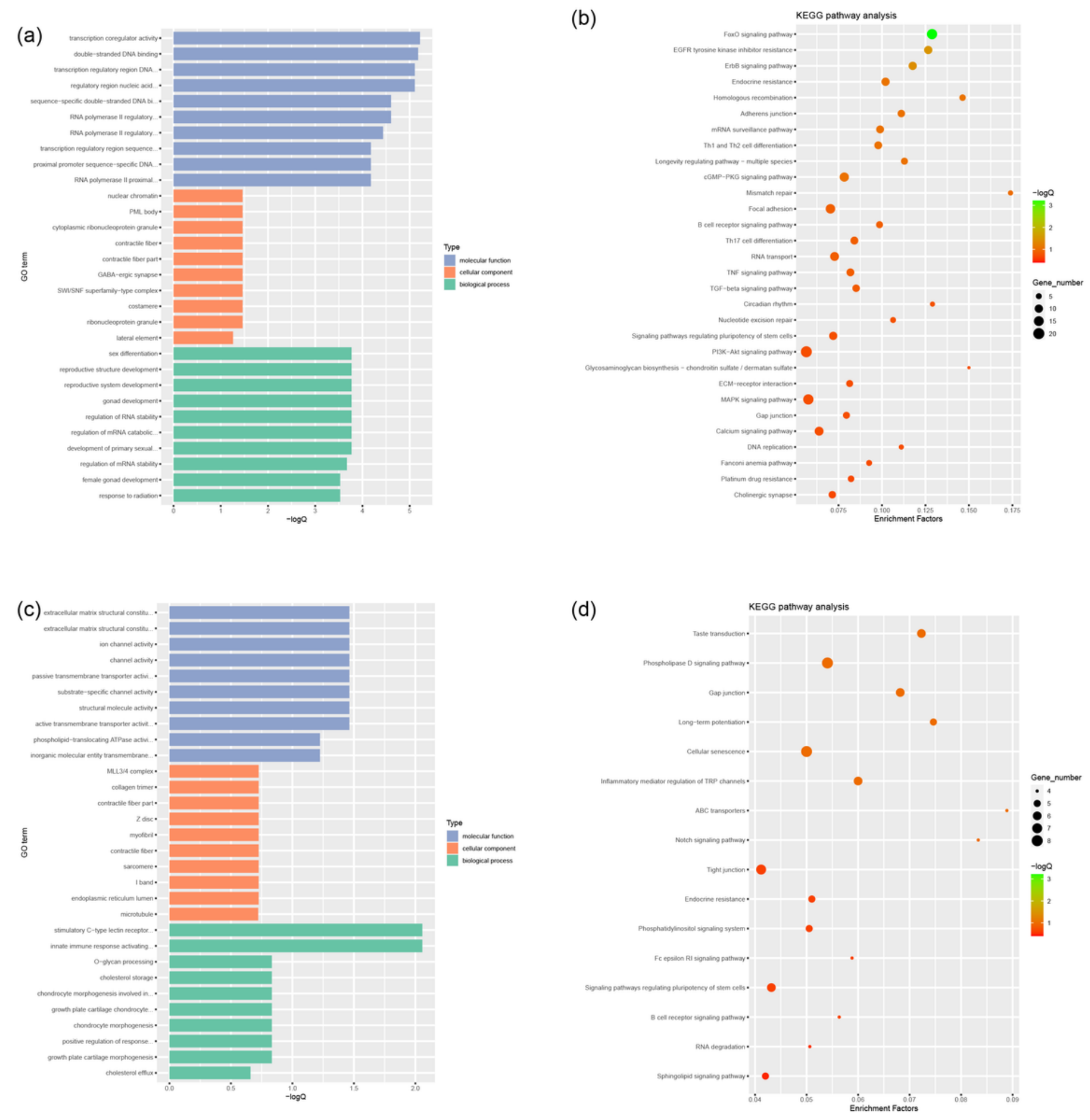

\section{Figure 4}

Pathway analysis for somatic mutations and germline mutations. (a, b) GO and KEGG pathway for somatic mutations. (c, d) GO and KEGG pathway for germline mutations.

\section{Supplementary Files}

This is a list of supplementary files associated with this preprint. Click to download. 
- S.Table1.MutationSummary.xIsx 